This is the final peer-reviewed accepted manuscript of:

Barletta, A. (2015). A proof that convection in a porous vertical slab may be unstable. Journal of Fluid Mechanics, 770, 273-288

The final published version is available online at:

https://doi.org/10.1017/ifm.2015.154

Rights / License:

The terms and conditions for the reuse of this version of the manuscript are specified in the publishing policy. For all terms of use and more information see the publisher's website.

This item was downloaded from IRIS Università di Bologna (https://cris.unibo.it/)

When citing, please refer to the published version. 


\title{
A proof that convection in a porous vertical slab may be unstable
}

\author{
A. Barletta $\dagger$ \\ Department of Industrial Engineering, Alma Mater Studiorum Università di Bologna, \\ Viale Risorgimento 2, 40136 Bologna, Italy
}

(Received 6 March 2015)

The stability of the natural convection parallel flow in a vertical porous slab is reconsidered, by reformulating the problem originally solved in Gill's classical paper of 1969 (Journal of Fluid Mechanics, vol. 35, pp. 545-547). A comparison is made between the set of boundary conditions where the slab boundaries are assumed to be isothermal and impermeable (Model A), and the set of boundary conditions where the boundaries are modelled as isothermal and permeable (Model B). It is shown that Gill's proof of linear stability for Model A cannot be extended to Model B. The question about the stability/instability of the basic flow is examined by carrying out a numerical solution of the stability eigenvalue problem. It is shown that, with Model B, the natural convection parallel flow in the basic state becomes unstable when the Darcy-Rayleigh number is larger than 197.081. The normal modes selected at onset of instability are transverse rolls. Direct numerical simulations of the nonlinear regime of instability are carried out.

\section{Introduction}

A stationary and parallel flow may exist in a vertical channel bounded by impermeable and isothermal parallel planes kept at different temperatures. This flow is caused by the action of the buoyancy force and is endowed with a zero vertical mass flow rate. Such a flow may be established, with different features, either for a fluid filling the channel or for a fluid saturated porous slab. In both cases, the temperature profile across the channel is linear, as in pure conduction, so that this buoyant flow regime is usually called conduction regime.

A cornerstone result in the analysis of the conduction regime for a vertical porous slab governed by Darcy's law was obtained by Gill (1969). This author, by employing a linear stability analysis, was able to prove that the stationary and parallel natural convection flow in a vertical porous slab is always stable. Despite the sharp conclusion drawn by Gill (1969), further investigations were carried out later on by other authors. The main motivation of these studies is the suggestion stated by Gill at the end of his paper that a possible instability could be onset by including inertial effects in the local momentum balance equation, i.e. by altering the classical formulation of Darcy's law. Rees (1988) reformulated Gill's stability problem by taking into account the term proportional to the time-derivative of velocity in the momentum balance. This author was able to carry out a very detailed and thorough numerical solution of the eigenvalue stability problem in order to determine the growth rates of the perturbations imposed on the basic flow. Rees (1988) concluded that, in spite of the improvement in the momentum balance formulation, Gill's conclusion that the conduction regime is always stable maintained its validity. Further results complementary to the analyses carried out by Gill (1969) and Rees (1988) were 
reported by Lewis et al. (1995), relative to a regime of very large Darcy-Rayleigh numbers. Other authors (Wolanski 1973; Straughan 1988; Flavin \& Rionero 1999) reconsidered Gill's stability problem by a fully nonlinear analysis. In particular, Straughan (1988) proved that nonlinear disturbances of the basic flow decay in time, at least exponentially, under suitable initial conditions. In other words, Straughan (1988) showed that Gill's proof of stability can be extended to the nonlinear domain of perturbations. Kwok \& Chen (1987) investigated separately the effect of no-slip boundary conditions for velocity, implemented by Brinkman's model of momentum balance instead of Darcy's law, and the effect of temperature-dependent viscosity within Darcy's law. These authors found that, within a linear stability analysis, both no-slip conditions and variable viscosity are able to yield instability and, hence, to modify the conclusion implied by Gill's proof. It must be mentioned that, in both cases examined by Kwok \& Chen (1987), the basic flow states differ from that considered by Gill (1969). Two very recent papers (Rees 2011; Scott \& Straughan 2013) present a new perspective on Gill's problem by taking into account the lack of local thermal equilibrium between fluid phase and solid phase. These authors confirmed the conclusion that the basic flow is stable with either linear (Rees 2011) or nonlinear (Scott \& Straughan 2013) stability analyses.

The aim of this paper is to prove that the role of the velocity boundary conditions may be very important in determining the onset of instability. The flow system considered and the mathematical model adopted are exactly the same as those assumed by Gill (1969). The only difference is in the velocity boundary conditions as the vertical boundaries are assumed to be permeable instead of impermeable. This change has no influence on the form of the basic natural convection flow, viz. the conduction regime, so that the basic state is exactly the same as that considered by Gill (1969). On the other hand, the conclusions drawn from the linear stability analysis are markedly different. The extent of this difference is assessed in two steps. First, Gill's proof is reconsidered to show that it cannot be extended to the case of permeable walls. Then, a numerical solution of the stability eigenvalue problem is employed to determine the conditions for the onset of instability. Finally, the nonlinear regime of instability is studied by direct numerical simulations carried out with a finite element solver of the governing equations.

\section{Mathematical model}

The subject of this analysis is the natural convection in a vertical porous slab bounded by two vertical planes at $x= \pm L / 2$ where uniform temperatures $T_{1}$ and $T_{2}$ are prescribed. The $x$-axis is horizontal and perpendicular to the bounding planes, the $y$-axis is vertical and oriented upward, while the $z$-axis is horizontal.

\subsection{Governing equations}

We model the fluid saturated porous medium through Darcy's law, by invoking the validity of the Oberbeck-Boussinesq approximation. Furthermore, we assume that local thermal equilibrium between the solid and the fluid exists and that no internal heat source is present. Thus, the governing equations can be written in a dimensionless form as

$$
\begin{gathered}
\boldsymbol{\nabla} \cdot \boldsymbol{u}=0, \\
\boldsymbol{u}=-\boldsymbol{\nabla} p+R T \hat{\boldsymbol{e}}_{y}, \\
\frac{\partial T}{\partial t}+\boldsymbol{u} \cdot \boldsymbol{\nabla} T=\nabla^{2} T,
\end{gathered}
$$




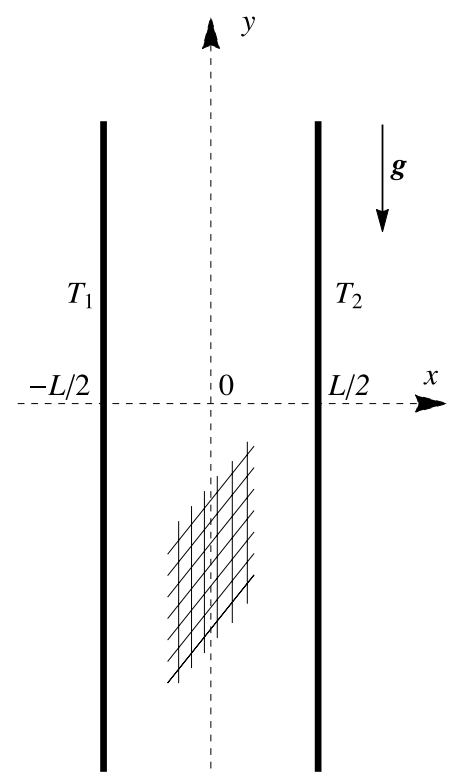

Model A

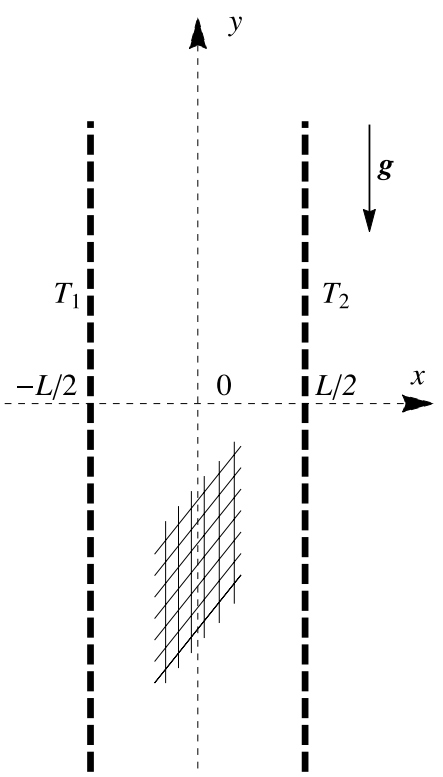

Model B

Figure 1. A sketch of Models A and B in terms of dimensional quantities

where $\hat{\boldsymbol{e}}_{y}$ is the unit vector along the $y$-axis. The dimensional coordinates $(x, y, z)$, time $t$, dynamic pressure $p$, seepage velocity $\boldsymbol{u}=(u, v, w)$, and temperature $T$ have been scaled to define the corresponding dimensionless quantities, namely

$$
\begin{aligned}
& \frac{1}{L}(x, y, z) \rightarrow(x, y, z), \quad \frac{\alpha}{\sigma L^{2}} t \rightarrow t, \quad \frac{K}{\mu \alpha} p \rightarrow p, \\
& \frac{L}{\alpha} \boldsymbol{u}=\frac{L}{\alpha}(u, v, w) \rightarrow(u, v, w)=\boldsymbol{u}, \quad \frac{T-T_{0}}{T_{2}-T_{1}} \rightarrow T .
\end{aligned}
$$

Here, $\alpha$ is the average thermal diffusivity, $\sigma$ is the ratio between the average volumetric heat capacity of the saturated porous medium and the volumetric heat capacity of the fluid, $K$ is the permeability, $\mu$ is the dynamic viscosity, and $T_{0}=\left(T_{1}+T_{2}\right) / 2$ is the reference temperature. The term dynamic pressure is used here to denote the local difference between the pressure and the hydrostatic pressure.

The Darcy-Rayleigh number $R$ is defined as

$$
R=\frac{g \beta\left(T_{2}-T_{1}\right) K L}{\nu \alpha},
$$

where $g$ is the modulus of the gravitational acceleration $\boldsymbol{g}=-g \hat{\boldsymbol{e}}_{y}$, while $\beta$ is the thermal expansion coefficient of the fluid, and $\nu$ is the kinematic viscosity of the fluid.

\subsection{Boundary conditions}

As sketched in Fig. 1 by employing dimensional quantities, we devise two possible sets of boundary conditions relative to the planes $x= \pm 1 / 2$. 


\subsubsection{Model $A$}

We can imagine these planes as isothermal and impermeable following Gill's approach (Gill 1969), namely

$$
x= \pm 1 / 2: \quad u=0, \quad T= \pm 1 / 2 .
$$

\subsubsection{Model B}

The alternative is to model the boundaries as permeable. In this case, the external environments in the regions $x<-1 / 2$ and $x>1 / 2$ are considered as isothermal fluid reservoirs in a motionless state and kept at different uniform temperatures, $T_{1}$ and $T_{2}$. In these reservoirs, the pressure distribution is purely hydrostatic, so that the dynamic pressure is zero. Hence, we formulate the boundary conditions

$$
x= \pm 1 / 2: \quad p=0, \quad T= \pm 1 / 2 .
$$

\subsubsection{Comparison between Models $A$ and $B$}

The importance for the building industry of Model A was pointed out by Gill (1969). A vertical porous slab with impermeable side boundaries, is conceived by this author as an effective method to produce an insulated gap within a building wall. In this context, the suppression of heat transfer enhancement, beyond pure conduction, is definitely a desired feature (Gill 1969). Model B converts the perfectly impermeable boundaries of the porous slab into perfectly permeable boundaries. A real insulation slab is more realistically modelled with imperfectly impermeable, or partly permeable, boundaries. This reasonably means a behaviour intermediate between Model A and Model B. On the other hand, Model B appears closer to systems such as the "breathing walls" (Imbabi 2006). Dynamic insulation and breathing walls are, in fact, novel concepts in the building industry aimed to achieve a better indoor air quality by allowing air inflow/outflow through insulated building walls.

As pointed out by Elder (1967), natural convection in a porous medium is important to geophysics, especially for the analysis of geothermal sites and hot-spring areas. This author also mentioned the phenomenon of mass discharge in the study of hydrothermal systems and of penetrative convection in the upper mantle. As in Model B, defined by Eq. (2.5), Elder (1967) employed a free boundary, considered as a surface at constant pressure, to model the natural mass discharge from a horizontal porous layer heated from below. For a survey and bibliography about more recent applications to geophysics of convection in porous media, the reader may refer, for instance, to Chapter 9 of the book by Turcotte \& Schubert (2014).

\section{Stability analysis}

By considering either Model A or Model B boundary conditions, a stationary solution of Eqs. (2.1) is given by

$$
u_{b}=0, \quad v_{b}=R x, \quad w_{b}=0, \quad T_{b}=x, \quad p_{b}=0,
$$

which is exactly the basic flow analysed by Gill (1969).

By perturbing the basic flow, Eq. (3.1), viz. by defining

$$
\boldsymbol{u}=\boldsymbol{u}_{b}+\varepsilon \tilde{\boldsymbol{u}}, \quad p=p_{b}+\varepsilon \tilde{p}, \quad T=T_{b}+\varepsilon \tilde{T},
$$

we may rewrite Eqs. (2.1), (2.4) and (2.5) in terms of the fields $\tilde{\boldsymbol{u}}=(\tilde{u}, \tilde{v}, \tilde{w}), \tilde{p}$ and $\tilde{T}$, 
namely

$$
\begin{array}{cc}
\boldsymbol{\nabla} \cdot \tilde{\boldsymbol{u}}=0, & \\
\tilde{\boldsymbol{u}}=-\boldsymbol{\nabla} \tilde{p}+R \tilde{T} \hat{\boldsymbol{e}}_{y}, & \\
\frac{\partial \tilde{T}}{\partial t}+R x \frac{\partial \tilde{T}}{\partial y}+\tilde{u}=\nabla^{2} \tilde{T}, & \\
x= \pm 1 / 2: \quad \tilde{u}=0, \quad \tilde{T}=0 \quad \text { Model A, } \\
x= \pm 1 / 2: \quad \tilde{p}=0, \quad \tilde{T}=0 \quad \text { Model B, }
\end{array}
$$

where terms of $O\left(\varepsilon^{2}\right)$ are neglected according to the usual linearisation procedure.

We now simplify Eqs. (3.3) by adopting a pressure/temperature formulation,

$$
\begin{array}{cc}
\nabla^{2} \tilde{p}=R \frac{\partial \tilde{T}}{\partial y}, & \\
\frac{\partial \tilde{T}}{\partial t}+R x \frac{\partial \tilde{T}}{\partial y}-\frac{\partial \hat{p}}{\partial x}=\nabla^{2} \tilde{T}, & \\
x= \pm 1 / 2: \quad \frac{\partial \tilde{p}}{\partial x}=0, \quad \tilde{T}=0 \quad \text { Model A }, \\
x= \pm 1 / 2: \quad \tilde{p}=0, \quad \tilde{T}=0 \quad \text { Model B. }
\end{array}
$$

The solution of Eqs. (3.4) is expressed in terms of normal modes, so that $\tilde{p}$ and $\tilde{T}$ are given by

$$
\tilde{p}=f(x) e^{i\left(k_{y} y+k_{z} z\right)} e^{(\eta-i \omega) t}, \quad \tilde{T}=h(x) e^{i\left(k_{y} y+k_{z} z\right)} e^{(\eta-i \omega) t} .
$$

Each normal mode is associated with a wave vector, $\left(k_{y}, k_{z}\right)$, a wave number, $k=\left(k_{y}^{2}+\right.$ $\left.k_{z}^{2}\right)^{1 / 2}$, a growth rate, $\lambda$, and an angular frequency, $\omega$. For the sake of brevity, we use the notation $\lambda=\eta-i \omega$. For the normal modes, Eqs. (3.4) yield

$$
\begin{gathered}
f^{\prime \prime}-k^{2} f-i k_{y} R h=0, \\
h^{\prime \prime}-\left(k^{2}+\lambda+i k_{y} R x\right) h+f^{\prime}=0, \\
x= \pm 1 / 2: \quad f^{\prime}=0, \quad h=0 \quad \text { Model A, } \\
x= \pm 1 / 2: \quad f=0, \quad h=0 \quad \text { Model B, }
\end{gathered}
$$

where primes denote derivatives with respect to $x$.

As suggested by Rees (1988), general oblique modes can be mapped onto transverse modes $\left(k_{z}=0\right)$ by defining a rescaled Darcy-Rayleigh number,

$$
S=\frac{k_{y}}{k} R,
$$

so that Eqs. (3.6) can be rewritten as

$$
\begin{gathered}
f^{\prime \prime}-k^{2} f-i k S h=0, \\
h^{\prime \prime}-\left(k^{2}+\lambda+i k S x\right) h+f^{\prime}=0, \\
x= \pm 1 / 2: \quad f^{\prime}=0, \quad h=0 \quad \text { Model A, } \\
x= \pm 1 / 2: \quad f=0, \quad h=0 \quad \text { Model B. }
\end{gathered}
$$

The limit $S \rightarrow 0$ thus defines longitudinal modes $\left(k_{y}=0\right)$. The conclusion that longitudinal modes are equivalent to transverse modes having a vanishing Darcy-Rayleigh 
number suggests that these modes are always stable. A formal proof that this argument is correct is provided in Section 3.2.

\subsection{Gill's proof revisited}

We derive Eq. (3.8a) with respect to $x$, obtain $f^{\prime}(x)$ from Eq. $(3.8 b)$ and substitute it into the derived Eq. (3.8a), so that we can write a single fourth-order differential equation for $h(x)$, namely

$$
\begin{aligned}
& h^{\prime \prime \prime \prime}-\left(2 k^{2}+\lambda\right) h^{\prime \prime}-i k S\left(x h^{\prime}\right)^{\prime}+k^{2}\left(k^{2}+\lambda+i k S x\right) h=0, \\
& x= \pm 1 / 2: \quad h=0, \quad h^{\prime \prime}=0 \quad \text { Model A, } \\
& x= \pm 1 / 2: \quad h=0, \quad h^{\prime \prime \prime}=\left(k^{2}+\lambda \pm i k S / 2\right) h^{\prime} \quad \text { Model B. }
\end{aligned}
$$

We now multiply Eq. (3.9a) by $\bar{h}$, the complex conjugate of $h$, and we integrate by parts over $-1 / 2<x<1 / 2$ using the boundary conditions $h( \pm 1 / 2)=0$, common to both Model A and Model B. Thus, we obtain

$$
\begin{gathered}
\bar{h}^{\prime}(-1 / 2) h^{\prime \prime}(-1 / 2)-\bar{h}^{\prime}(1 / 2) h^{\prime \prime}(1 / 2)+\int_{-1 / 2}^{1 / 2}\left|h^{\prime \prime}\right|^{2} d x+\left(2 k^{2}+\lambda\right) \int_{-1 / 2}^{1 / 2}\left|h^{\prime}\right|^{2} d x \\
+i k S \int_{-1 / 2}^{1 / 2} x\left(\left|h^{\prime}\right|^{2}+k^{2}|h|^{2}\right) d x+k^{2}\left(k^{2}+\lambda\right) \int_{-1 / 2}^{1 / 2}|h|^{2} d x=0 .
\end{gathered}
$$

Since $\lambda=\eta-i \omega$, the real part of Eq. (3.10) gives

$$
\gamma+\int_{-1 / 2}^{1 / 2}\left(\left|h^{\prime \prime}\right|^{2}+2 k^{2}\left|h^{\prime}\right|^{2}+k^{4}|h|^{2}\right) d x=-\eta \int_{-1 / 2}^{1 / 2}\left(\left|h^{\prime}\right|^{2}+k^{2}|h|^{2}\right) d x
$$

where

$$
\gamma=\mathfrak{R e}\left[\bar{h}^{\prime}(-1 / 2) h^{\prime \prime}(-1 / 2)-\bar{h}^{\prime}(1 / 2) h^{\prime \prime}(1 / 2)\right] .
$$

If Model $\mathrm{A}$ is adopted, then Eq. (3.9b) ensures that $\gamma=0$, so that Eq. (3.11) allows one to conclude that $\eta<0$. This means that the growth rate of normal modes is always negative or, in other words, that the basic flow given by Eq. (3.1) is always stable. This conclusion completes the classical Gill's proof of stability (Gill 1969).

The important point is that there is no evidence that Gill's proof can be extended to Model B. In fact, for Model B, the boundary conditions given by Eq. (3.9b) do not allow one to draw any direct conclusion about $\gamma$ being negative, zero or positive. Hence, Gill's method to prove stability becomes ineffective in this case.

\subsection{Numerical solution}

The lack of a formal proof of stability for Model B leaves an open possibility to investigate the stability or instability of the basic flow, Eq. (3.1), through a numerical solution of the eigenvalue problem given by Eqs. (3.8).

This problem can be solved with a high numerical accuracy by combining the use of an initial value solver for ordinary differential equations and the shooting method. This mathematical technique is well documented in the literature (see, for instance, $\S 19.1$ of the book by Straughan (2004)). The procedure can be outlined in two steps: 
(a) We transform the boundary value problem into an initial value problem by replacing Eq. (3.8c) with

$$
f(-1 / 2)=0, \quad f^{\prime}(-1 / 2)=\xi_{1}+i \xi_{2}, \quad h(-1 / 2)=0, \quad h^{\prime}(-1 / 2)=1 .
$$

The last condition, $h^{\prime}(-1 / 2)=1$, is meant to fix the otherwise arbitrary scale of the eigenfunctions, while $\left(\xi_{1}, \xi_{2}\right)$ are unknown real constants to be determined by the shooting method.

By prescribing the values of $(k, S)$ and trying to guess the values of $\left(\eta, \omega, \xi_{1}, \xi_{2}\right)$, the initial value problem given by Eqs. $(3.8 a),(3.8 b)$ and (3.13) can be solved by an explicit Runge-Kutta algorithm.

(b) The shooting method is employed to compute the values of $\left(\eta, \omega, \xi_{1}, \xi_{2}\right)$ versus the input values $(k, S)$ by satisfying the target conditions given by Eq. (3.8c), namely $f(1 / 2)=0$ and $h(1 / 2)=0$. The eigenfunctions $f$ and $h$ are complex-valued, so that the target conditions yield four real equations.

Both steps can be accomplished by using Mathematica 10 (c) Wolfram Research), with function NDSolve providing the environment for step 1 and function FindRoot yielding the algorithm to implement step 2.

The most interesting quantity to be computed is $\eta$, whose values can be traced, for a fixed $k$, as $S$ increases starting from 0 . In fact, the solution of Eqs. (3.8) when $S \rightarrow 0$ can be obtained analytically. When $S \rightarrow 0$, Eq. (3.8a) with the boundary conditions $(3.8 c)$ for Model B admits only the solution $f=0$, so that $h$ can be determined by solving

$$
\begin{gathered}
h^{\prime \prime}-\left(k^{2}+\eta-i \omega\right) h=0, \\
x= \pm 1 / 2: \quad h=0 .
\end{gathered}
$$

The eigenfunctions are

$$
h(x)=\frac{1}{n \pi} \sin \left[n \pi\left(x+\frac{1}{2}\right)\right], \quad n=1,2,3, \ldots,
$$

with the dispersion relation

$$
k^{2}+\eta-i \omega+n^{2} \pi^{2}=0, \quad n=1,2,3, \ldots .
$$

Thus, on remembering that $f=0$, Eqs. (3.15) and (3.16) yield

$$
\eta=-k^{2}-n^{2} \pi^{2}, \quad \omega=0, \quad \xi_{1}=0, \quad \xi_{2}=0, \quad n=1,2,3, \ldots .
$$

Equation (3.17) provides the initialisation of the numerical procedure, through steps 1 and 2 described above. With these steps, one evaluates $\left(\eta, \omega, \xi_{1}, \xi_{2}\right)$ for any given $k$ and gradually increasing values of $S$ starting from $S=0$.

As expected from Eq. (3.17), the values of the growth rate $\eta$ are initially negative, when $S$ is sufficiently small. The condition $\eta<0$ means stability. However, if $S$ becomes large enough also positive values of $\eta$ are found, meaning instability. As $k$ changes, one can draw the geometrical locus of the points where $\eta=0$ in the parametric plane $(k, S)$. This locus is the neutral stability curve and it maps the boundary of the instability region.

Table 1 reports a validation of the numerical procedure, for $k=1$ and $S=10$. The assessment of the accuracy is made by comparing the numerical data $\left(\eta, \omega, \xi_{1}, \xi_{2}\right)$ obtained by using either a fixed step-size specification in the explicit fourth-order RungeKutta solver or an adaptive method for variable step-size. The data reported are relative to the branch of normal modes displaying the largest growth rates, $\eta$. On decreasing monotonically the value of the step-size $\delta x$, Table 1 shows that convergence to the data obtained with the adaptive method is attained within at least seven significant figures. 


$\begin{array}{ccrcc}\delta x & \eta & \omega & \xi_{1} & \xi_{2} \\ 1 / 10 & -6.5720827111 & 0.0115230246 & 3.6666762654 & -5.4721318872 \\ 1 / 20 & -6.5812277719 & 0.0003740971 & 3.6615896896 & -5.4712607706 \\ 1 / 30 & -6.5818525481 & 0.0000495981 & 3.6613072452 & -5.4712226333 \\ 1 / 40 & -6.5819621832 & 0.0000117975 & 3.6612595920 & -5.4712166593 \\ 1 / 50 & -6.5819925973 & 0.0000038700 & 3.6612465761 & -5.4712150850 \\ 1 / 60 & -6.5820036004 & 0.0000015562 & 3.6612419052 & -5.4712145317 \\ 1 / 70 & -6.5820083361 & 0.0000007202 & 3.6612399047 & -5.4712142980 \\ 1 / 80 & -6.5820106401 & 0.0000003695 & 3.6612389345 & -5.4712141858 \\ 1 / 90 & -6.5820118683 & 0.0000002051 & 3.6612384185 & -5.4712141265 \\ 1 / 100 & -6.5820125710 & 0.0000001211 & 3.6612381238 & -5.4712140929 \\ 1 / 200 & -6.5820138298 & 0.0000000038 & 3.6612375974 & -5.4712140333 \\ \text { adaptive } & -6.5820138691 & -0.0000000063 & 3.6612375868 & -5.4712140314\end{array}$

TABLE 1. Validation of the numerical solver: comparison between values of $\left(\eta, \omega, \xi_{1}, \xi_{2}\right)$ obtained, for $k=1$ and $S=10$, by an explicit fourth-order Runge-Kutta solver with either fixed step-size, $\delta x$, or variable step-size determined adaptively
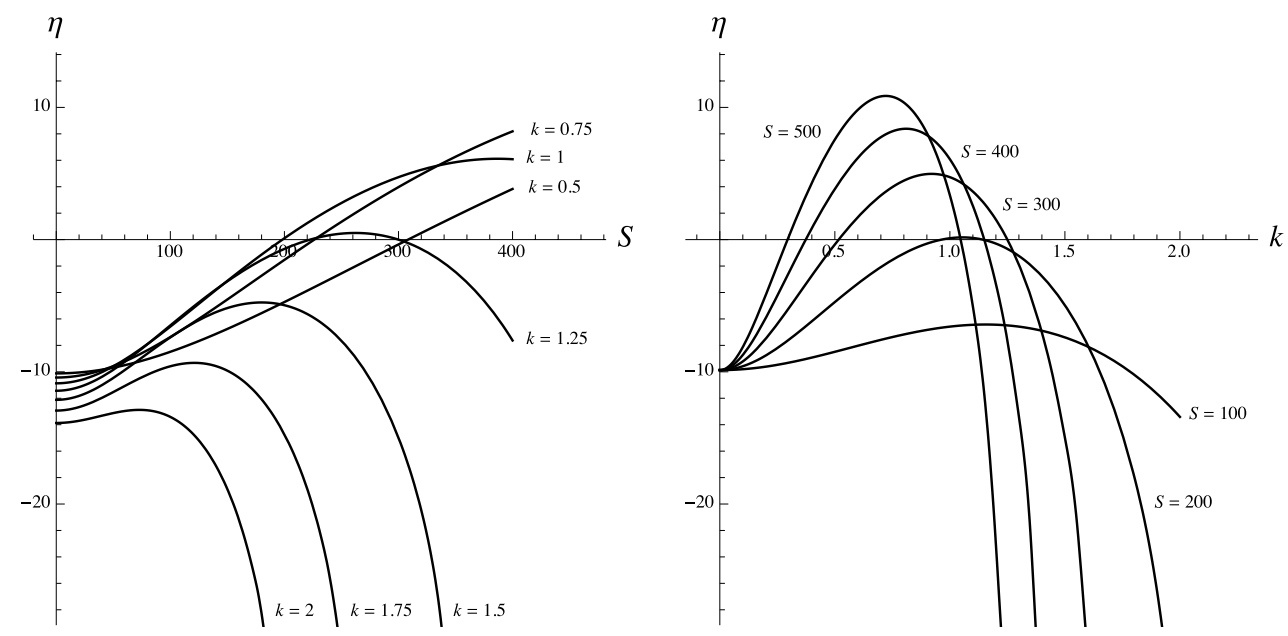

Figure 2. Model B: growth rate $\eta$ versus $S$ for different wave numbers $k$ (left frame); growth rate $\eta$ versus $k$ for different $S$ (right frame)

The values of $\omega$ need a specific mention, as the numerical data suggest that $\omega$ is effectively zero, meaning that the normal mode is non-travelling. All the graphical and numerical results reported in the forthcoming section are obtained by the explicit fourth-order Runge-Kutta solver with an adaptive method for variable step-size.

\section{Discussion of the results}

\subsection{Linear analysis}

In order to establish the possible onset of unstable conditions in the parametric plane $(k, S)$, we first study the change of the growth rate $\eta$ versus $S$ for fixed values of $k$.

Growth rate plots are reported in Fig. 2. As for Table 1, we examine data relative to the branch with the largest growth rates. The left frame of Fig. 2 displays plots of $\eta$ versus $S$ for fixed values of $k$, while the right frame shows plots of $\eta$ versus $k$ for fixed values of $S$. 

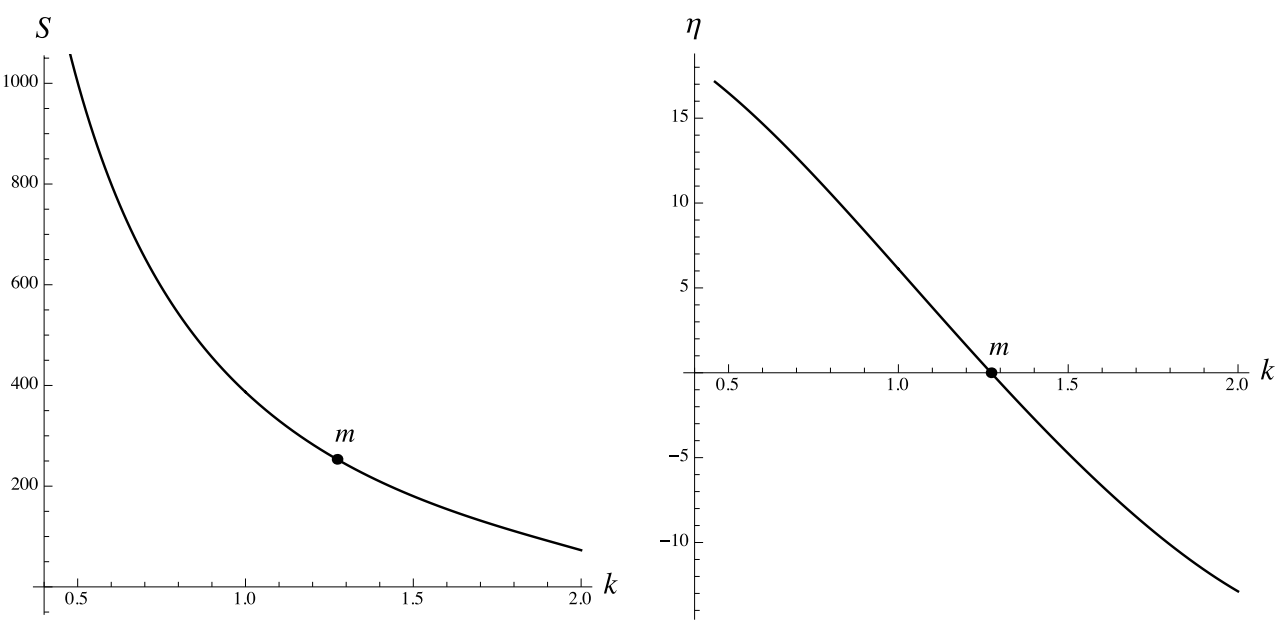

Figure 3. Model B: Line of maximum growth rate, for fixed $k$, in the plane $(k, S)$ (left frame) and in the plane $(k, \eta)$ (right frame). Point $m$ denotes conditions where the maximum growth rate is zero, namely $k_{m}=1.27291$ and $S_{m}=253.340$
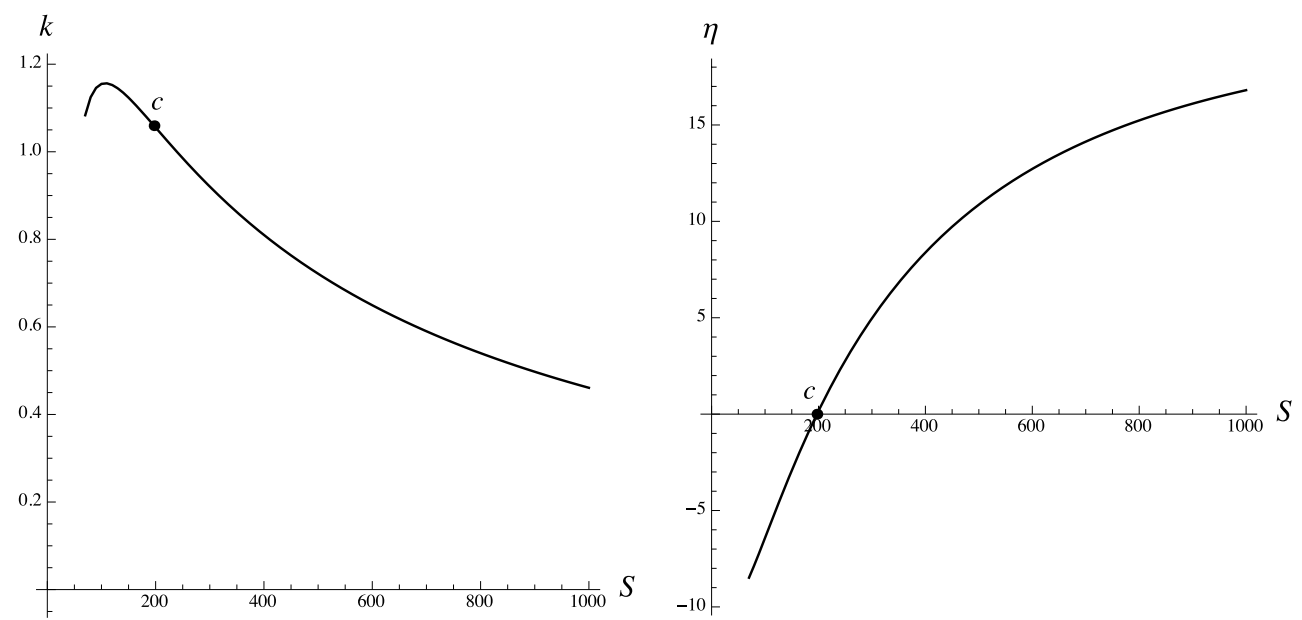

Figure 4. Model B: Line of maximum growth rate, for fixed $S$, in the plane $(S, k)$ (left frame) and in the plane $(S, \eta)$ (right frame). Point $c$ denotes conditions where the maximum growth rate is zero, namely $k_{c}=1.05950$ and $S_{c}=197.081$

In the left frame of Fig. 2, wave numbers ranging from 0.5 to 2 are considered. Starting from $k=0.5$, we note that the growth rate $\eta$ starts from a negative value when $S=0$ (longitudinal rolls), then gradually increases and becomes positive when $S$ is greater than 308.427. The change of sign occurs with a smaller $S$ if $k=0.75$, and with an even smaller $S$ when $k=1$. The displayed behaviour is different if we consider $k=1.25$ or larger values of $k$. In fact, with $k=1.25$, the growth rate $\eta$ is initially negative and, as $S$ increases, it becomes positive within the range $222.588<S<299.943$, then it becomes negative again and starts decreasing. This non-monotonic trend of $\eta$ versus $S$ is observed also with larger wave numbers, $k=1.5,1.75,2$, but the growth rate does not become positive in these cases. A common feature of the data reported in Fig. 2 is that 

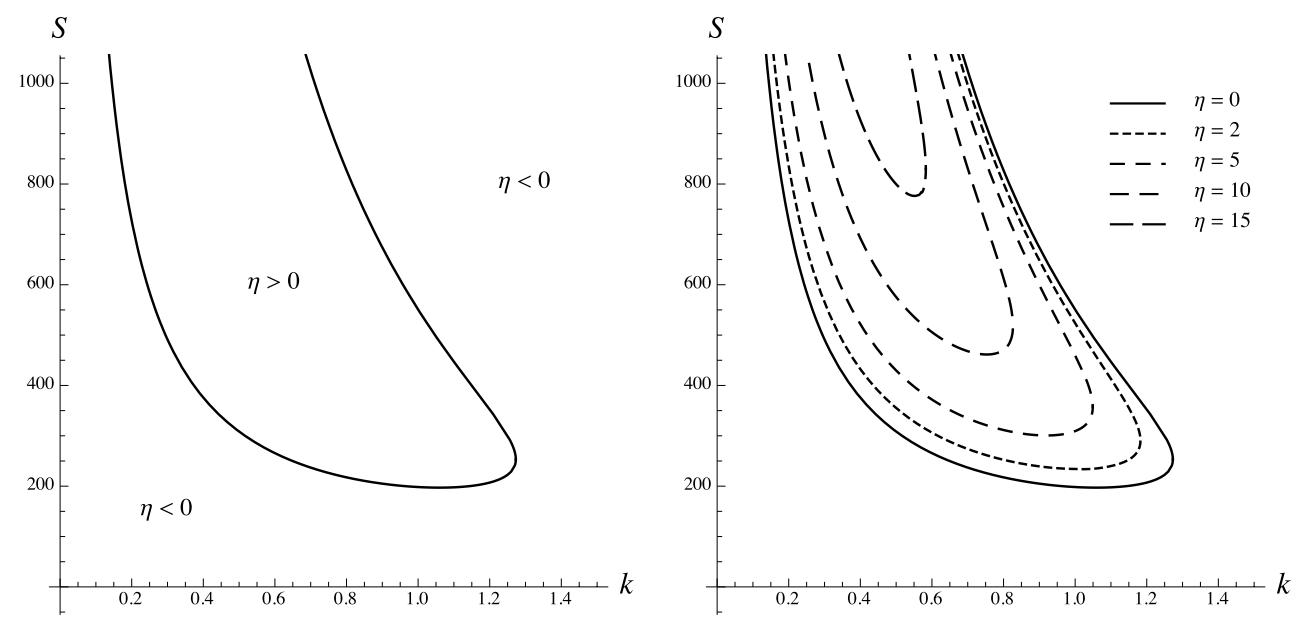

Figure 5. Model B: neutral stability curve $S$ versus $k$ (left frame) and isolines of $\eta$ with $\eta \geqslant 0$ in the plane $(k, S)$ (right frame)

the value of $\omega$ is zero, i.e. they are relative to non-travelling modes. We mention that this feature is not general as branches of stable travelling modes $(\omega=0)$ arise outside the range reported in Fig. 2, where $\eta$ is lower than -30 . The existence of stable travelling modes had been previously reported by Rees (1988) with reference to Model A boundary conditions. Figure 2 suggests the existence of a maximum growth rate $\eta$ as a function of $S$ for a given $k$. Likewise, there exists a maximum of function $\eta(k)$ for a given $S$. The conditions which yield the maximum growth rate $\eta(S)$ for a fixed $k$, as well as those for the maximum growth rate $\eta(k)$ for a fixed $S$, are reported in Figs. 3 and 4 . Both these figures show the pairs $(k, S)$, or $(S, k)$, of maximum growth rate, as well as the maximum growth rate plotted either versus $k$ or versus $S$. Figures 3 and 4 show also the points, $m$ and $c$, where the maximum of function $\eta(S)$ is zero and the maximum of function $\eta(k)$ is zero, respectively. These figures are useful to detect the fastest growing mode when either the wavenumber is prescribed (Fig. 3) or the parameter $S$ is prescribed (Fig. 4). The importance of the fastest growing linear mode in determining the development of convective instability has been recently pointed out by Hewitt et al. (2013).

The left frame of Fig. 5 shows the neutral stability curve $(\eta=0)$, as well as the stability $(\eta<0)$ and instability $(\eta>0)$ regions in the plane $(k, S)$. The right frame of Fig. 5 shows isolines of $\eta$ including not only the neutral stability curve $(\eta=0)$, but also some isolines with positive $\eta$.

All neutrally stable modes are non-travelling, viz. $\omega=0$. The neutral stability curve has a nose shape, and it displays a minimum at the critical conditions $k=k_{c}$ and $S=S_{c}$, where

$$
k_{c}=1.05950, \quad S_{c}=197.081 .
$$

Figure 5 shows also the existence of a maximum value of $k$ bounding the neutrally stable states, this maximum defines the tip of the nose and is placed at

$$
k_{m}=1.27291, \quad S_{m}=253.340 .
$$

One can immediately realise that the pairs $(k, S)$ defined by Eqs. (4.1) and (4.2) correspond to the points $c$ and $m$ displayed in Figs. 4 and 3, respectively. No unstable modes 

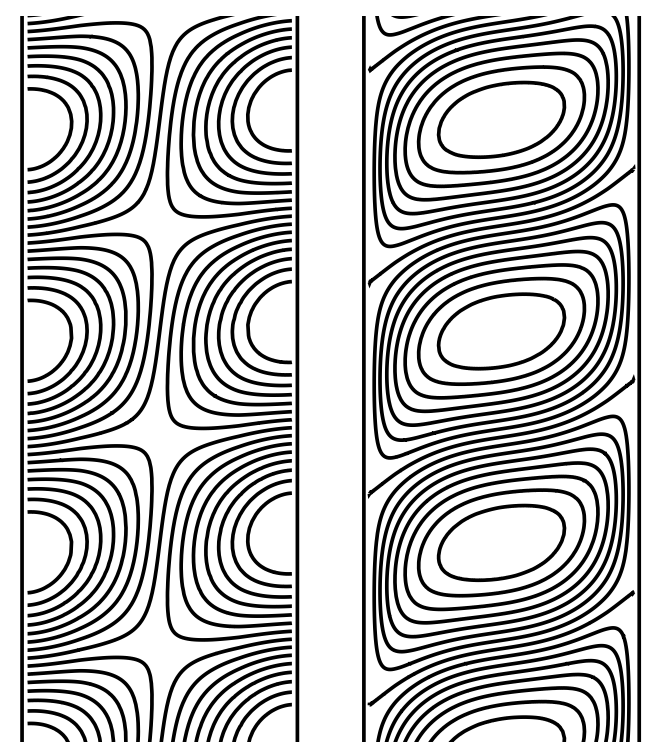

Figure 6. Model B: streamlines (left frame) and isotherms (right frame) of the secondary flow induced by transverse modes at onset of instability with the critical values $\left(k_{c}, S_{c}\right)$, Eq. (4.1)

could be found when $k>k_{m}$. Due to the definition of $S$, given by Eq. (3.7), the critical value $S_{c}$ means that instability has its onset with transverse modes $\left(k_{y}=k\right)$ at a critical Darcy-Rayleigh number $R_{c}=197.081$. Oblique modes $\left(k_{y}<k\right)$ are destabilised at higher critical Darcy-Rayleigh numbers, obtained by dividing $S_{c}$ by the ratio $k_{y} / k$. This yields larger and larger critical values of $R$ as $k_{y} / k$ decreases starting from 1 . Eventually, in the limiting case of longitudinal modes $\left(k_{y} \rightarrow 0\right)$, one obtains $R_{c} \rightarrow \infty$, which means stability to this type of modes. In other words, Squire's theorem selecting the two-dimensional transverse modes at onset of instability holds.

An example of a system where the instability defined by the critical conditions (4.1) manifests itself may come from geophysics. Let us consider a gravel layer with thickness $L=2 \mathrm{~m}$ and permeability $K=5 \times 10^{-10} \mathrm{~m}^{2}$, saturated with groundwater, $\nu=10^{-6} \mathrm{~m}^{2} / \mathrm{s}$, $\alpha=10^{-7} \mathrm{~m}^{2} / \mathrm{s}$ and $\beta=2 \times 10^{-4} \mathrm{~K}^{-1}$. Instability, $R>R_{c}$, occurs when the temperature difference across the layer is larger than $10 \mathrm{~K}$.

A sketch of the streamlines and isotherms for the secondary flow arising at onset of convection with $k_{c}=1.05950$ and $R_{c}=197.081$ is shown in Fig. 6 . Due to the expression of normal modes, Eq. (3.5), the convection pattern forms a periodic sequence of alternate clockwise and counterclockwise cells, so that arrows are not strictly needed in order to understand the orientation of streamlines in Fig. 6. As discussed above, this secondary flow is through non-travelling transverse modes. One may see that the streamlines are cut by the boundaries, so that the secondary flow penetrates both the left external environment $(x<-1 / 2)$ and the right external environment $(x>1 / 2)$. Thus, one may question about the reliability of Model B at supercritical conditions, where the growing disturbances achieve finite or large amplitudes. At supercritical conditions, fancying the external environments as quiescent reservoirs at uniform temperature and hydrostatic pressure distribution may be a poor model. In fact, the penetrating secondary flow is likely to induce inhomogeneities of the temperature and dynamic pressure distributions therein. 

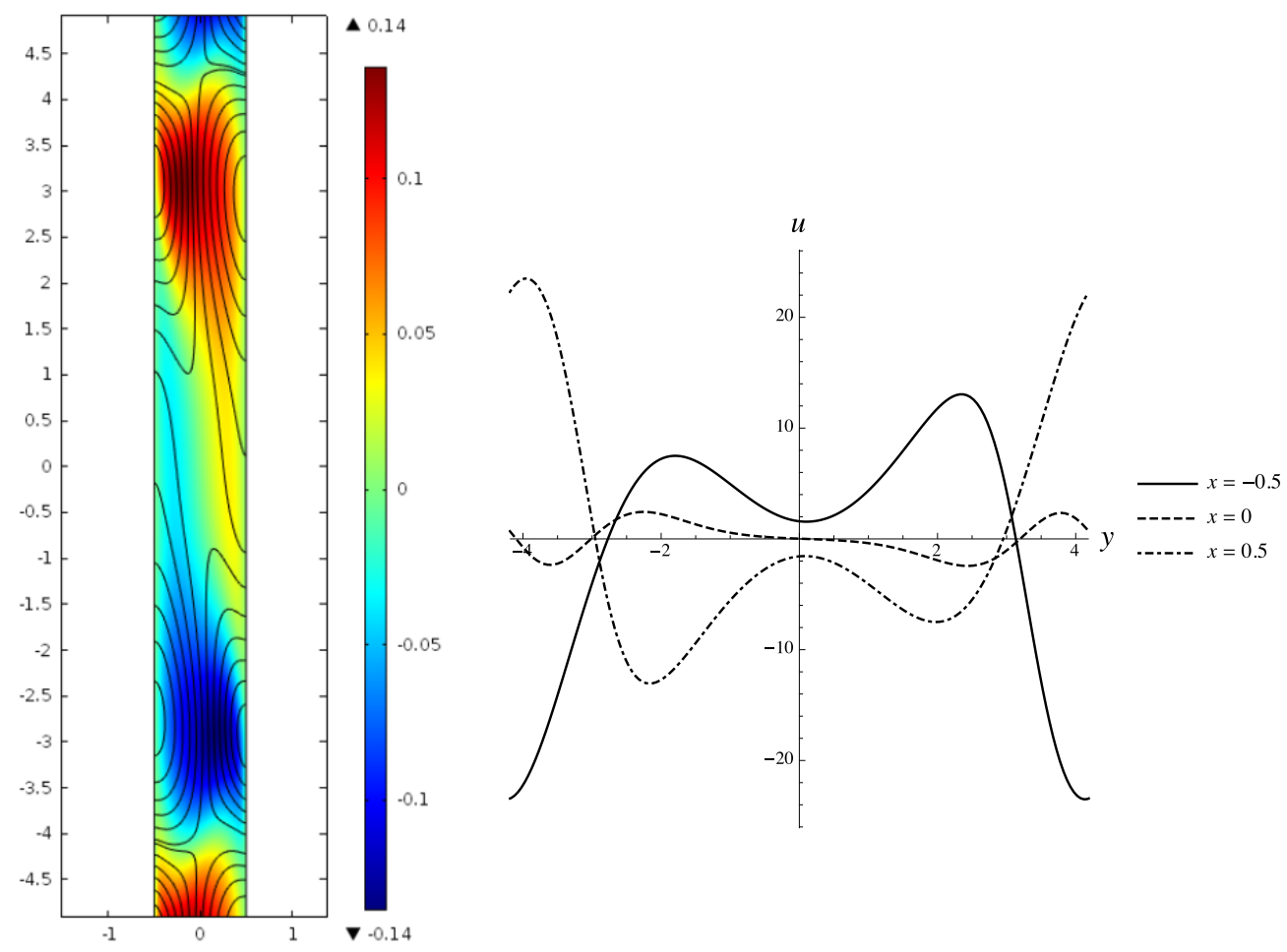

FIgURE 7. Asymptotic steady state for $t \rightarrow \infty$ in the nonlinear regime with $R=400$ and $\Delta y=8 \pi$ : colour map over one period of the temperature perturbation with superposed streamlines of the velocity perturbation in the $(x, y)$-plane (left frame); plot over one period of the horizontal velocity component $u$ as a function of $y$ at different fixed values of $x$ (right frame)
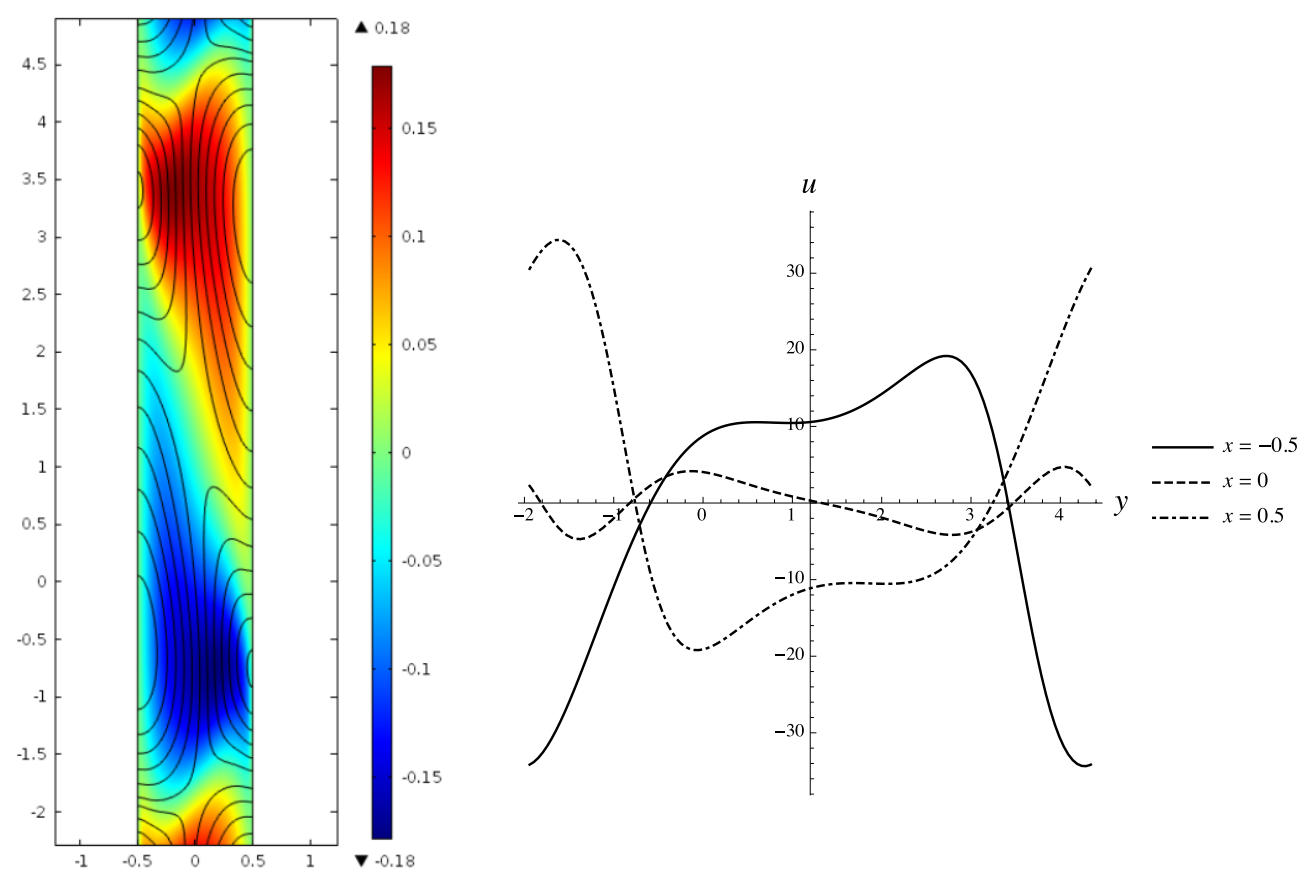

FiguRE 8. Asymptotic steady state for $t \rightarrow \infty$ in the nonlinear regime with $R=400$ and $\Delta y=4 \pi$ : colour map over one period of the temperature perturbation with superposed streamlines of the velocity perturbation in the $(x, y)$-plane (left frame); plot over one period of the horizontal velocity component $u$ as a function of $y$ at different fixed values of $x$ (right frame) 


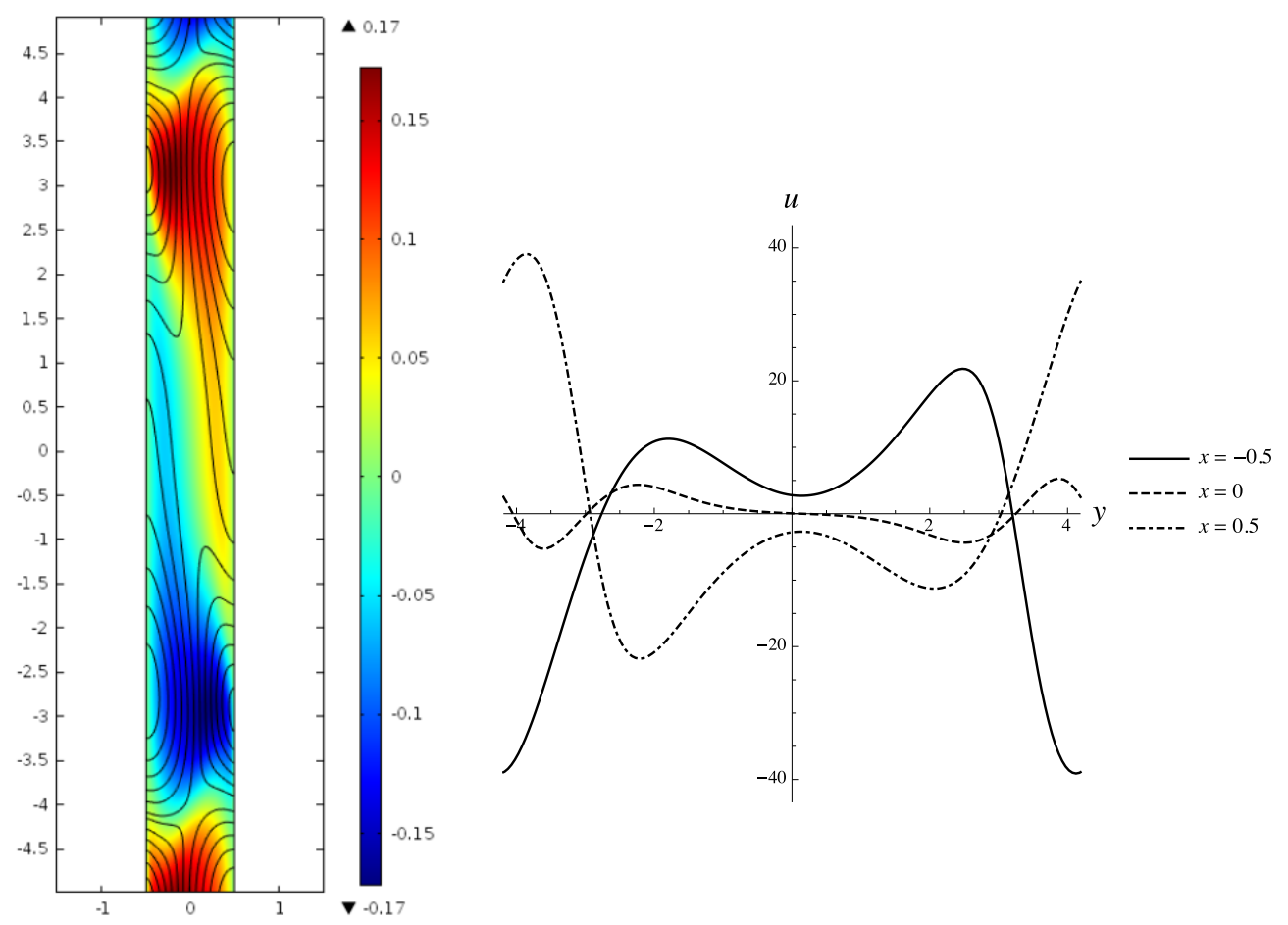

Figure 9. Asymptotic steady state for $t \rightarrow \infty$ in the nonlinear regime with $R=500$ and $\Delta y=8 \pi$ : colour map over one period of the temperature perturbation with superposed streamlines of the velocity perturbation in the $(x, y)$-plane (left frame); plot over one period of the horizontal velocity component $u$ as a function of $y$ at different fixed values of $x$ (right frame)
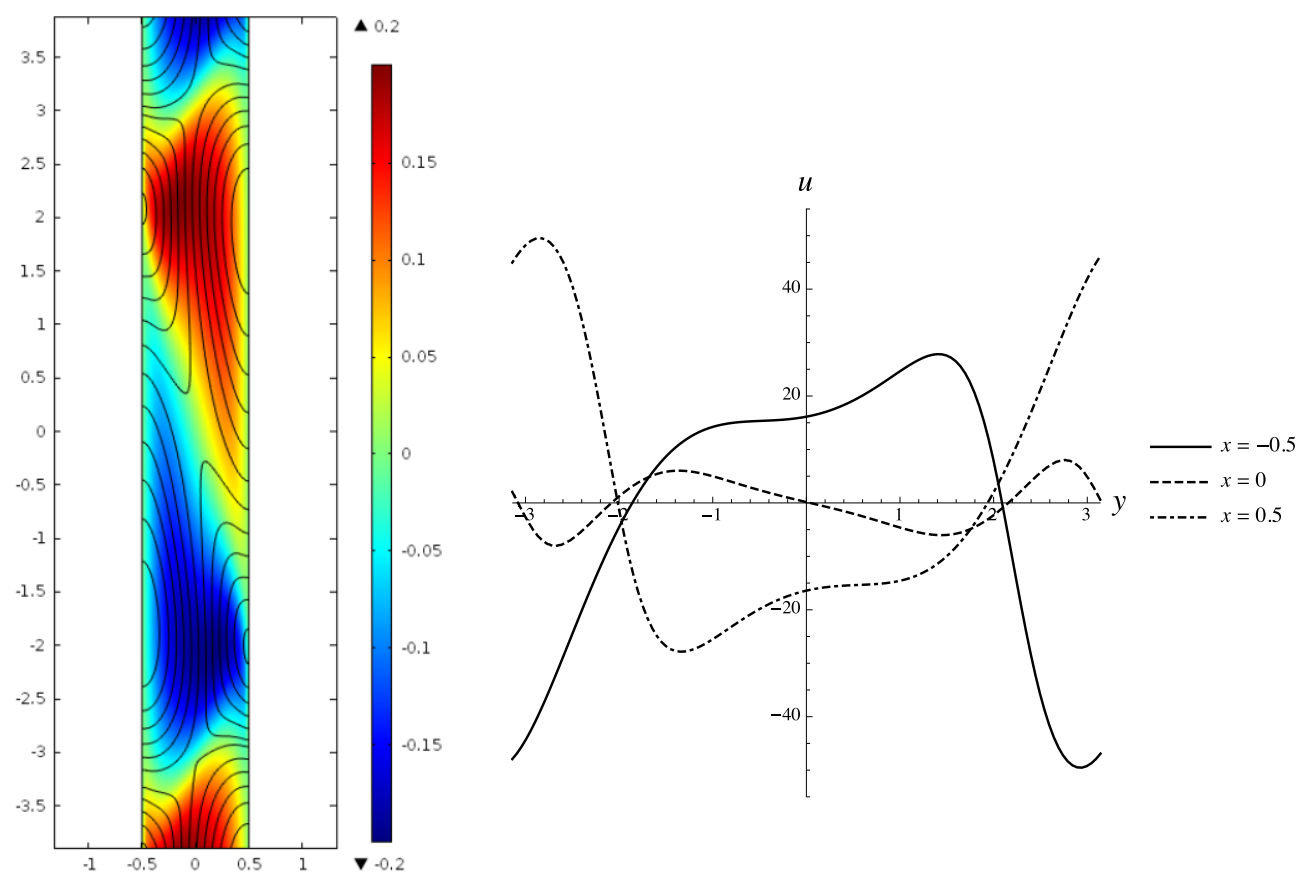

Figure 10. Asymptotic steady state for $t \rightarrow \infty$ in the nonlinear regime with $R=500$ and $\Delta y=4 \pi$ : colour map over one period of the temperature perturbation with superposed streamlines of the velocity perturbation in the $(x, y)$-plane (left frame); plot over one period of the horizontal velocity component $u$ as a function of $y$ at different fixed values of $x$ (right frame) 


\subsection{A few examples of nonlinear regime}

When the flow conditions are supercritical, i.e. $R>R_{c}$, a nonlinear analysis is needed to trace the time evolution of finite-amplitude perturbations. This means that the full nonlinear governing equations (2.1) and (2.5) have to be solved. The solution can be sought numerically by employing a finite element method. An efficient tool for achieving this solution is COMSOL Multiphysics (c) COMSOL Inc.). The solution can be carried out with reference to transverse modes, so that the computational domain is two-dimensional. The domain defined for the solver is a rectangle in the $(x, y)$-plane with $-1 / 2 \leqslant x \leqslant 1 / 2$ and $-\Delta y / 2 \leqslant y \leqslant \Delta y / 2$. In the solution of the full nonlinear equations, the role of normal modes is different from that in the linear analysis. In fact, the nonlinear time evolution of a normal mode with wave number $k$, prescribed at time $t=0$, implies its mixing with all possible normal modes. Another aspect of the nonlinear evolution of unstable perturbations is the phenomenon of saturation. Saturation means that the initially exponential time-growth of the perturbation is dampened, so that the growth-rate decreases and it may even tend to 0 as $t \rightarrow \infty$. In this case, the unbounded exponential growth predicted by the linear stability analysis is drastically modified in the nonlinear domain, thus leading to the prediction of asymptotic stationary states, different from the basic state, approached at large times. The phenomenon of saturation may also lead to the conclusion that the effects of instability are not always so remarkable as one would infer from the linear stability analysis. Before discussing a few sample cases in the supercritical regime, it is worth describing some details of the numerical solution. Periodicity conditions for pressure and temperature are prescribed at the end boundaries, $y=-\Delta y / 2$ and $y=\Delta y / 2$. An unstructured uniform mesh with triangular elements is defined in the computational domain. Two cases are studied with $\Delta y=4 \pi$ and $\Delta y=8 \pi$. With $\Delta y=4 \pi$ the number of triangular elements is 12630 , while for $\Delta y=8 \pi$ the number of elements is 25184 . The mesh refinement has been chosen so that the numerical results are mesh-independent within graphical resolution. In the four sample cases analysed, the time evolution tends to a stationary state different from the basic state.

The four cases illustrated in Figs. 7-10 are relative to $R=400$ and $R=500$ and, for each fixed value of $R$, they describe different patterns along the vertical $y$-direction. In fact, we assigned different values of $\Delta y$, namely $\Delta y=8 \pi$ and $\Delta y=4 \pi$, to explore different periods of the cellular patterns. The region plotted in the left frames of Figs. 710 is not the whole range of $y$, but approximately one period. In these frames, the colour map of the temperature perturbation, viz. the difference between the temperature and the temperature in the basic state, is plotted together with the streamlines of the perturbation velocity, namely the excess velocity with respect to the basic state. The right frames of Figs. 7-10 display the horizontal velocity component, $u$, as a function of $y$ for $x=-1 / 2,0,1 / 2$. We recall that $u=0$ in the basic state, so that this velocity component arises from the perturbation of the basic state. In the four cases examined, the streamlines evidence a natural mass discharge/recharge to/from the external environments. Likewise the phenomenon reported in the paper by Elder (1967), the lateral mass flux is not a very intense effect, both for $R=400$ and for $R=500$. In fact, the maximum values of $u$ at the lateral boundaries are slightly greater than 20 (Fig. 7) or 30 (Fig. 8), when $R=400$, and around 40 (Fig. 9) or slightly greater (Fig. 10), when $R=500$. A reference value to estimate these maximum values of $u$ is the maximum vertical velocity in the basic state, that is $R / 2$. This means 200, for Figs. 7 and 8, and 250, for Figs. 9 and 10 . The temperature perturbation is comparable with the basic state temperature, as the maxima in the colour maps are not much less than half the maximum temperature in 
the basic state, namely $T=1 / 2$. A final remark regards the periodicity of the patterns as related to the prescribed values of $\Delta y$. One may notice that, when $\Delta y=8 \pi$ (Figs. 7 and 9 ), three periods are allowed over the height of the computational domain. On the other hand, when $\Delta y=4 \pi$ (Figs. 8 and 10), two periods are allowed. With reference to the case $R=400$, there is a simple physical argument justifying this pattern selection. In fact, over a height of $8 \pi$ with periodicity end conditions, allowed normal modes are such that $k=n / 4$, where $n=1,2,3, \ldots$. Over a height of $4 \pi$, allowed normal modes are with $k=n / 2$. The right frame of Fig. 2 reveals that, with $R=400$, the linear mode of type $k=n / 4$ with the largest growth rate is for $n=3$, while the fastest growing mode with $k=n / 2$ is for $n=2$. This explains why the 3 -periods pattern is preferred for a height of $8 \pi$, while the 2-periods pattern is preferred for a height of $4 \pi$. On the contrary, this reasoning does not work properly when $R=500$. In fact, by employing the data reported in the right frame of Fig. 2, the linear mode of type $k=n / 4$ with the largest growth rate is again for $n=3$, but the fastest growing mode with $k=n / 2$ is for $n=1$. Therefore, one should expect that a 1-period pattern is selected when the height is $4 \pi$, unlike what is found by the nonlinear analysis. An explanation of the ineffectiveness of the fastest growing linear mode in determining the periodicity of the actual nonlinear pattern is related to the highly supercritical regime taking place at $R=500$. In this regime, the nonlinear dynamics prevails so that the fastest growing linear mode is unable to drive the pattern selection mechanism.

The nonlinear analysis in the supercritical regime carried out so far has definitely no aim of generality. There is much more work to do for an exhaustive investigation of this regime. An open question is, for instance, the multiplicity of periodic cellular structures that are allowed in the asymptotic steady states approached when $t \rightarrow \infty$. In this regard, the role of the end conditions set at the extrema of the $y$-range needs further investigation. In particular, an interesting point is the description of what may happen if the periodicity end conditions, at $y=-\Delta y / 2$ and $y=\Delta y / 2$, are replaced by impermeability and thermal insulation conditions. This alternative may be appropriate in order to model a vertical slab with a finite height.

\section{Conclusions}

The linear stability of the conduction regime in a vertical porous slab saturated by a fluid is reconsidered on the basis of a different formulation of the velocity boundary conditions. The boundaries are assumed to be permeable to external environments in motionless states with a hydrostatic pressure distribution. This assumption replaces the hypothesis of impermeable boundaries invoked in other classical treatments of the same flow regime (Gill 1969). The basic natural convection flow, stationary and parallel, is not influenced by the altered boundary conditions. On the other hand, it has been proved that the linear stability analysis changes dramatically as a consequence of the different model for the boundaries. The extent of this change has been assessed in two stages:

- Gill's proof of stability, for the case of impermeable boundaries, has been restated without forcing any assumption about the velocity boundary conditions. Thus, it has been evidenced where this proof may fail if the boundaries are considered as permeable instead of impermeable.

- A numerical solution of the eigenvalue stability problem has been employed to obtain the growth rates of the three-dimensional perturbations superposed to the basic flow. It is shown that, on increasing the value of the Darcy-Rayleigh number, the growth rates turn from negative (stability) to positive (instability). A suitable Squire-like transformation allowed us to identify the transverse modes as the selected modes at onset of instability. 
The neutral stability curve has been obtained. Its minimum at a Darcy-Rayleigh number equal to 197.081 yields the critical condition for the onset of instability with transverse modes.

- A few examples have been discussed where the full nonlinear governing equations have been solved to investigate the supercritical regime, $R>R_{c}$. Saturation of the growth rates has been pointed out, showing that spatially-periodic steady states different from the basic state can be approached at large times. In these steady states, natural mass discharge to the external fluid environments is observed, even if the local values of velocity normal to the free boundaries are definitely smaller than the basic vertical velocity.

This study has shown the dramatic effect of a change in the velocity boundary conditions on the stability of the basic flow in a vertical porous slab. Modifying the boundaries from impermeable to permeable implies that the same basic state, instead of being always stable, may become unstable.

\section{Acknowledgements}

This research has been carried out with the financial support provided by Alma Mater Studiorum Università di Bologna with FARB and FIBRA grants.

\section{REFERENCES}

ELDER, J. W. 1967 Steady free convection in a porous medium heated from below. Journal of Fluid Mechanics 27, 29-48.

Flavin, J. N. \& Rionero, S. 1999 Nonlinear stability for a thermofluid in a vertical porous slab. Continuum Mechanics and Thermodynamics 11, 173-179.

Gill, A. E. 1969 A proof that convection in a porous vertical slab is stable. Journal of Fluid Mechanics 35, 545-547.

Hewitt, D. R., Neufeld, J. A. \& Lister, J. R. 2013 Stability of columnar convection in a porous medium. Journal of Fluid Mechanics 737, 205-231.

IMBABI, M. S.-E. 2006 Modular breathing panels for energy efficient, healthy building construction. Renewable Energy 31, 729-738.

Kwok, L. P. \& CHEN, C. F. 1987 Stability of thermal convection in a vertical porous layer. ASME Journal of Heat Transfer 109, 889-893.

Lewis, S., Bassom, A. P. \& Rees, D. A. S. 1995 The stability of vertical thermal boundarylayer flow in a porous medium. European Journal of Mechanics B Fluids 14, 395-407.

ReEs, D. A. S. 1988 The stability of Prandtl-Darcy convection in a vertical porous layer. International Journal of Heat and Mass Transfer 31, 1529-1534.

REEs, D. A. S. 2011 The Effect of Local Thermal Nonequilibrium on the Stability of Convection in a Vertical Porous Channel. Transport in Porous Media 87, 459-464.

Scott, N. L. \& Straughan, B. 2013 A Nonlinear Stability Analysis of Convection in a Porous Vertical Channel Including Local Thermal Nonequilibrium. Journal of Mathematical Fluid Mechanics 15, 171-178.

Straughan, B. 1988 A nonlinear analysis of convection in a porous vertical slab. Geophysical E6 Astrophysical Fluid Dynamics 42, 269-275.

Straughan, B. 2004 The Energy Method, Stability, and Nonlinear Convection, 2nd edn. New York, NY: Springer.

Turcotte, D. L. \& Schubert, G. 2014 Geodynamics, 3rd edn. Cambridge, UK: Cambridge University Press.

Wolanski, E. J. 1973 Convection in a vertical porous slab. Physics of Fluids 16, 2014-2016. 